\title{
Damping Mechanism of SiC Particle Reinforced Al Matrix Composites
}

\author{
Xiao LI, Chunmei LI, Dongyuan SHAO, Jingjing CHEN, \\ Qun HUI and Nanpu CHENG* \\ Faculty of Materials and Energy, Southwest University, Chongqing 400715, China
}

\begin{abstract}
The low-frequency internal friction behavior of $\mathrm{SiC}$ particle reinforced $\mathrm{Al}$ matrix composites was studied over a temperature range from $23^{\circ} \mathrm{C}$ to $550^{\circ} \mathrm{C}$ at frequencies of $0.1,1$ and $5 \mathrm{~Hz}$. The experimental data were analyzed in terms of the K-G-L dislocation theory and the anelastic relaxation of grain boundary sliding. Two internal friction peaks of the composites were respectively observed over the temperature ranges of $100 \sim 250^{\circ} \mathrm{C}$ and $200 \sim 500^{\circ} \mathrm{C}$. The dislocation motion is the important damping mechanism of the composites at low temperatures, while the grain boundary relaxation plays a dominant role at high temperatures. The activation energies for dislocation relaxation and grain boundary sliding are $1.2 \mathrm{eV}$ and $1.57 \mathrm{eV}$, respectively.
\end{abstract}

\section{Introduction}

$\mathrm{SiC}$ particle reinforced $\mathrm{Al}$ matrix composites $(\mathrm{SiCp} / \mathrm{Al})$ have great potentials to be used in aircrafts, vehicles, weapons and electronic devices, as high-temperature structural materials because of their enhanced damping capacity, high specific strength and stiffness together with the substantial retention of modulus and strength at elevated temperatures[1-4]. Efforts have been made on measurements of the internal friction in Al matrix composites with discontinuous reinforcements through deformation in a small strain range[5]. The basic elastic behavior of the materials remains to be further investigated especially when the temperature and strain-rate effects on deformation are considered. Such rate dependent elastic behavior is clearly not the ideal elasticity but the anelasticity that can be depicted by a linear differential strain-stress relation, in which an energy dissipative process is involved[6,7]. When examined in a dynamic experiment, the modulus in this linear stress-strain regime is regarded as $E^{*}=E_{1}+i E_{2}$, and the real and imaginary parts of which represent the storage and loss of mechanical energy, respectively. Correspondingly, the absolute value of $E^{*}, \sqrt{E_{1}^{2}+E_{2}^{2}}=\left|E^{*}\right| \equiv E$, and the ratio $E_{2} / E_{1}=\tan \phi$ represent the absolute elastic modulus and the internal friction, respectively.

\footnotetext{
*Corresponding author:cheng_np@swu.edu.cn
} 
In this work, the internal friction the $12 \% \mathrm{SiCp} / \mathrm{AA} 6066$ composite and the unreinforced alloy was studied at different vibration frequencies with continuous temperature scanning to understand the possible mechanisms, which in turn assists in preparation and design of the composite.

\section{Materials and Experimental Procedure}

The studied materials were prepared by a powder metallurgy route[8]. Fig.1 shows the microstructures of the as-received unreinforced alloy and composite, respectively. The grain size $(\sim 5 \mu \mathrm{m})$ of metal matrix in the composite is smaller than that of the alloy $(\sim 12 \mu \mathrm{m})$, and the dislocation density of the former is much greater than that of the latter. It also shows that the $\mathrm{SiC} / \mathrm{Al}$ interface is clean and well-knitted, and that some dispersoids formed along dislocations, whose subsequent motion will be inhibited by these fine particles. The samples of $2 \mathrm{~mm}$ in thickness, $4 \mathrm{~mm}$ in width and $70 \mathrm{~mm}$ in length were used in the internal friction measurement performed in a DMA machine at different vibration frequencies during thermal cycles between $300 \mathrm{~K}$ and $823 \mathrm{~K}$ at a heating rate of $2 \mathrm{~K} / \mathrm{min}$.

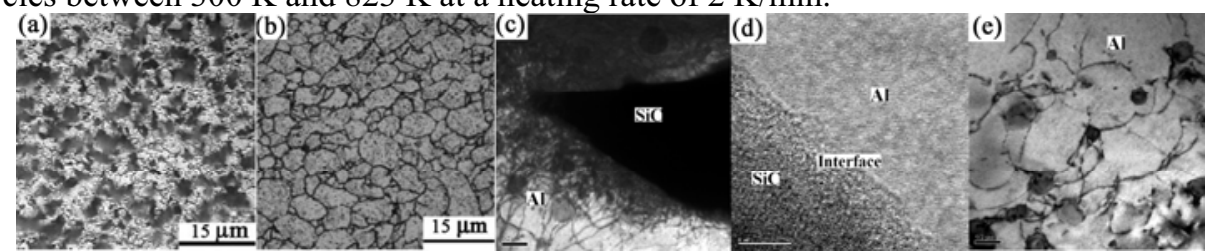

Fig.1 Microstructures of the test materials (a) OM of the composites; (b) OM of unreinforced alloy; (c) Dislocations in the composites; (d) a typical $\mathrm{SiC} / \mathrm{Al}$ interface; (e) subgrain in matrix

\section{Experimental Results}

Figs.2(a) shows the amplitude dependence of internal friction of the composite and the unreinforced alloy at room temperature and $1 \mathrm{~Hz}$. There is no internal friction peak to be found over the tested stress amplitude. The internal friction of the unreinforced matrix alloy increases slowly with the stress amplitude increasing, while that of the composite is larger and exhibits an exponential growth of the stress amplitude beyond a critical value ( $\varepsilon_{\text {crit }} \approx 2 \times 10^{-5}$ ). When $\varepsilon<1.5 \times 10^{-5}$, the values of internal friction are almost independent of the stress amplitude. Fig.2(b) gives an image of vibration frequency dependence of internal friction of the composite at room temperature and $\varepsilon=2.0 \times 10^{-5}$. It was found that the internal friction increases with the frequency increasing, and when $f<1 \mathrm{~Hz}$, the value of internal friction $Q^{-1} \propto f^{2}$, while $f>1 \mathrm{~Hz}, Q^{-1} \propto f$. Figs.2(c) and $(\mathrm{d})$ show the internal friction of the unreinforced alloy and the composite for different vibration frequencies with changing temperatures, respectively. In Fig.2(c), each $Q^{-1}$ vs $T$ curve of the unreinforced alloy exhibits a "hump" over a temperature range from 250 to $500{ }^{\circ} \mathrm{C}$ (the high temperature peak: HT peak), and ascends quickly when $T>500{ }^{\circ} \mathrm{C}$. In Fig.2(d), there also exhibits a similar hump in each of the $Q^{-1}$ vs $T$ curves for the composites. The "hump" in a curve is the presence of internal friction peak. In order not to omit the details of internal friction, the obtained data in the low temperature ranges are enlarged, and a similar hump is found in each $Q^{-1}$ vs $T$ curve for the composite (the low temperature peak: LT peak). However, no humps have been observed in curves for the unreinforced alloy in the low temperature ranges (see Fig.2(c)). All of these humps tend toward the low temperature side as the vibration frequency is lowered. The internal friction of the low frequency is lower than that of the high frequency at low temperatures, while it is the opposite at the high temperatures. 

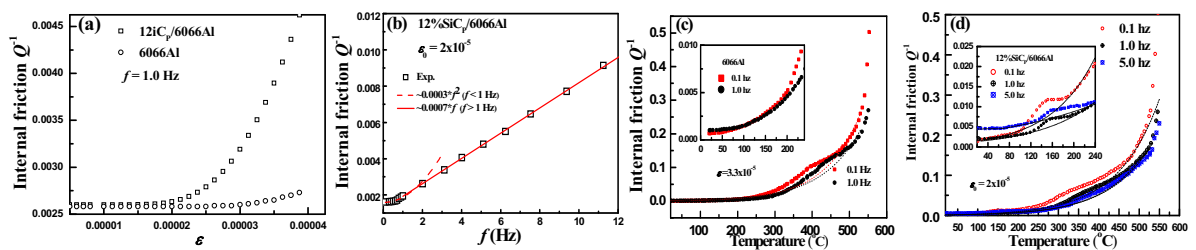

Fig.2 Internal friction of (a) composite and unreinforced alloy at $T=300 \mathrm{~K}$ and $f=1 \mathrm{~Hz}$;(b) composite at room temperature and $\varepsilon=2.0 \times 10^{-5} ;(\mathrm{c})$ matrix alloy and (d) composite at different frequencies

The humps appearing on the $Q^{-1}$ vs $T$ curves for the composite at low temperatures do not show up for the comparison materials without SiC particles. Thus, the LT peak is naturally ascribed to the presence of $\mathrm{SiC}$ reinforcements, whereas the $\mathrm{Al}$ matrix itself is responsible for the HT peak. To investigate the internal friction data, the "back ground" $\tan \phi_{\mathrm{B}}$ which originates from defects in the materials and becomes greater with increasing temperature should be subtracted[9]. Figs.3(a) and (b) show the background-subtracted internal friction of the matrix alloy and the composite, respectively. Now, the internal friction peaks appearing in Figs.3 (a) and (b) are clear and normally distributed, and it is easy to determine the peak temperatures $\left(T_{\mathrm{p}}\right)$ that are summarized in the Table 1.

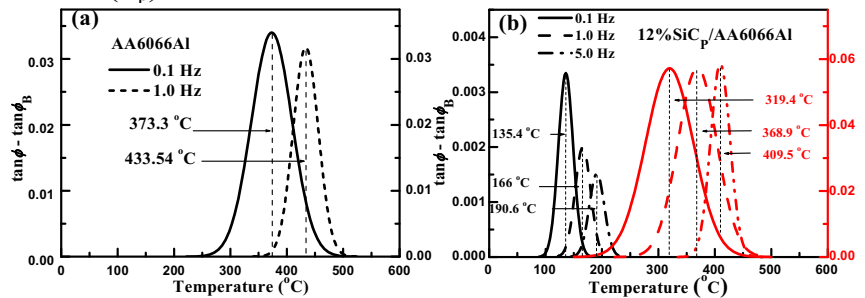

Fig. 3 The background subtracted internal friction (a)Al alloy and (b) the composite

Table 1 Peak Temperatures For The Unreinforced Alloy And The Composite

\begin{tabular}{|c|c|c|c|}
\hline Frequency(Hz) & AA6066 & \multicolumn{2}{|c|}{ Composite } \\
\hline & HT peak & LT peak & HT peak \\
\hline 0.1 & 646.5 & 408.6 & 529.6 \\
1 & 706.7 & 439.2 & 642.0 \\
5 & - & 463.8 & 682.7 \\
\hline
\end{tabular}

\section{Discussion}

In this work, one internal friction peak is observed over the range from 250 to $500{ }^{\circ} \mathrm{C}$ in the un-reinforced alloy. A similar peak is also observed over the same range in the composite, while another peak appears over the low temperature range. Based on the K-G-L theory, the dislocation amplitude-dependent internal friction at low temperatures can be written as[10,11]

$$
Q^{-1}=Q_{\mathrm{a}}^{-1}+Q_{\mathrm{b}}^{-1}=c_{1} \rho / \varepsilon_{0} \exp \left(-c_{2} / \varepsilon_{0}\right)+c_{3} \rho f
$$

Where $c_{1}, c_{2}$ and $c_{3}$ are three constants, $\rho$ is the dislocation density, $\varepsilon_{0}$ is the strain amplitude and $f$ is the vibration frequency, respectively. In Eq.(1), the internal friction is proportional to the dislocation density. The first term relies on the strain amplitude and is independent of the vibration frequency $f$, while the second one depends linearly on the vibration frequency $f$ and has no correlation to the strain amplitude $\varepsilon_{0}$. For a given $f$, 
$\ln \left(\varepsilon_{0} Q_{\mathrm{a}}^{-1}\right)=\ln \left(\varepsilon_{0} Q^{-1}-\varepsilon_{0} Q_{b}^{-1}\right)$ is linear to $1 / \varepsilon_{0}$, while for a given $\varepsilon_{0}$, $Q_{\mathrm{b}}^{-1}=Q^{-1}-Q_{\mathrm{a}}^{-1}=c_{3} \rho f$ is proportional to $f$. But, it is difficult to simultaneously determine the three constants, and some artifices should be employed. So, one can get

$$
\ln \left(\varepsilon_{0} Q_{\mathrm{a}}^{-1}\right)=\ln \left(\varepsilon_{0} \int \frac{\partial Q^{-1}}{\partial \varepsilon_{0}} \mathrm{~d} \varepsilon_{0}\right)=c_{1}-c_{2} / \varepsilon_{0}
$$

Fig.4 shows the experimental (the experimental data also shown in Fig.2) and theoretical results. It can be found that $\ln \left(\varepsilon_{0} Q_{\mathrm{a}}^{-1}\right)$ is linear to $1 / \varepsilon_{0}$ when the strain amplitude $\varepsilon_{0}>\varepsilon_{\text {crit }} \approx 2.0 \times 10^{-5}$, and the experimental results deviate from the theoretical ones when $\varepsilon_{0}<1.5 \times 10^{-5}$. The deviation means that the contribution of the vibration frequency can not be ignored at small amplitudes.

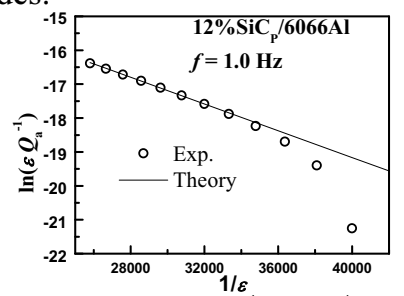

Fig.4 Correlation between $\ln \left(\varepsilon_{0} Q_{a}^{-1}\right)$ and $1 / \varepsilon_{0}$

An analogous treatment in mathematics for the experimental results plotted in Fig.2(b). It indicates that, for a given strain amplitude, the internal friction is linear to the vibration frequency when $f>1 \mathrm{~Hz}$, although the experimental results is proportional to $f^{2}$ when $f>1 \mathrm{~Hz}$. Torisaka has proved that the correlation between internal friction and vibration frequency conforms to $\ln Q^{-1} \propto n \ln f$, where $n$ is an empirical constant[12]. The above analysis has proved that the experimental results at low temperature coincide well with the K-G-L theory. In other words, dislocations play the dominant role in the internal friction of the composite at low temperature. The work of Zeners and Kê has revealed that a polycrystalline material shows anelastic relaxation due to the viscous nature of a grain boundary[6,13]. Once a viscous sliding occurs along a grain boundary in an elastically strained polycrystal subjected to a very small applied stress at high temperatures, the sliding motion between two adjacent grains gradually slows down because the neighboring grains block the sliding and the resulting elastic constraint becomes stronger as the sliding proceeds. The important observations of this phenomenon stem from Kê's elaborate work on Al, which provides an experimental foundation to the materials ranging from pure Al to solid solutions[13]. Since the matrix of $\mathrm{SiCp} / \mathrm{Al}$ composites, as well as the unreinforced $\mathrm{Al}$ alloy, is polycrystalline, the same relaxation phenomenon in these materials can be expected. From the experimental results and theoretical analysis, the internal friction peaks are caused by relaxation phenomenon, and the dislocations play important roles at low temperatures whereas the grain boundary sliding dominates the relaxation process at high temperatures. The relaxation time can be obtained by $\tau=\tau_{0} \exp \left(E / k_{\mathrm{B}} T\right)$. For the case of dislocation damping, the re-exponential factor conforms to $\tau_{0} \propto k_{\mathrm{B}} T$, therefore $\tau=c k_{\mathrm{B}} T \exp \left(E / k_{\mathrm{B}} T\right)$, where $c$ is a ratio but a function of frequency. An assumption has been made that the grain boundary relaxation time also satisfies the same correlation. The peak condition is 
$\omega \tau=\omega c k_{\mathrm{B}} T_{\mathrm{p}} \exp \left(E / k_{\mathrm{B}} T_{\mathrm{p}}\right)=\sqrt{1+\Delta_{\mathrm{E}}} \quad$ which can be transformed
into $\ln \left(\omega k_{\mathrm{B}} T_{\mathrm{p}}\right)=\ln \left(\sqrt{1+\Delta_{\mathrm{E}}} / c\right)-\left(E / k_{\mathrm{B}}\right) \cdot\left(1 / T_{\mathrm{p}}\right)$, where $T_{\mathrm{p}}$ is the peak temperature. The theoretical results are plotted in Fig.5.

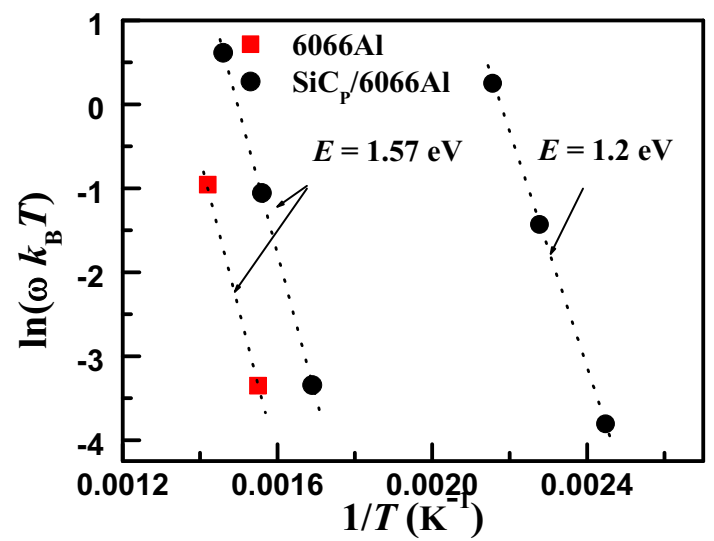

Fig. 5 The activation energies of LT and HT internal friction peaks

In Fig.5, the activation energies of the LT- and HT-peak of the composites are $1.2 \mathrm{eV}$ and $1.57 \mathrm{eV}$, respectively. The activation energy of the HT-peak of the unreinforced Al alloy is same to that of the composites, which reveals that the grain boundary relaxation is the common mechanism for the two materials at high temperature. However, the peak temperature of the composite at high temperature, compared with that of the unreinforced alloy, will decrease due to the refined grains with higher diffraction energy and more diffraction channels[6].

Another contribution to the internal friction of composites is the interface moving or sliding between metal matrix and reinforcements at high temperatures. Gu and Zhang have pointed out that if the temperature is higher than $100^{\circ} \mathrm{C}$, the interface sliding becomes possible or the microplastic deformation occurs in matrix near the interface under low stress due to the softened metal matrix[10]. This means that the interface damping should contribute to the internal friction of the composite. Given that the reinforcements are spherical and the interfacial cohesion is strong, Lavernia [12] has obtained the form of interfacial internal friction as $Q^{-1}=4.5 / \pi^{2} V_{\mathrm{P}}(1-v) /(2-v)$, where $V_{\mathrm{p}}$ and $v$ are the volume fraction and Poisson ratio of particle reinforcements, respectively. Because the Poisson ratio is insensitive to temperature, the interfacial internal friction is nearly determined by the volume fraction and is the order of $10^{-2}$ which coincides with the experimental results in the current work.

Practically, the relaxation of dislocations or grain boundaries in the tested materials is a multistage process. The dislocation density, the length of dislocation lines and the interaction between dislocation lines and point defects in different places are different. Hence, for the grain boundaries, the grain size is not uniform, and the size and the viscidity coefficient of grain boundaries are not same. All these features cause the multistage process in relaxation and form the broadened temperature ranges in the experiment. The grain refining can shift the relaxation peaks to an earlier time, but it will not change the activation energy of grain boundary relaxation (also see Fig.5). 


\section{Conclusions}

To examine the internal friction of the $12 \% \mathrm{SiCp} / 6066 \mathrm{Al}$ composite and the unreinforced Al alloy, a sub-resonant dynamic testing was carried out on a DMA apparatus. The data of measurements have been obtained over the range of 23 to $550^{\circ} \mathrm{C}$ at the frequencies of $0.1,1$ and $5 \mathrm{~Hz}$ with the strain amplitude kept at the order of $10^{-5}$. Two peaks of internal friction were found in the $12 \% \mathrm{SiCp} / 6066 \mathrm{Al}$ composite, i.e. the LT-peak over the 80 to $250^{\circ} \mathrm{Crange}$ and the HT-peak over the 250 to $500^{\circ} \mathrm{C}$ range, whereas only the HT-peak was clearly observed in the unreinforced $\mathrm{Al}$ alloy. With the vibration frequency increasing, all peaks shift toward the low temperature direction. The LT-peak in the composite is caused by the high-density dislocations which stems from the mismatches of thermal conductivity and elastic modulus between the metal matrix and the reinforcements, while the HT-peaks in the composite and the unreinforced alloy are created by the grain boundary relaxation. The activation energies for the LT- and HT-peak phenomena are $1.2 \mathrm{eV}$ and $1.57 \mathrm{eV}$, respectively. The theoretical analysis generally supports the description of these relaxation processes.

\section{Acknowledgements}

Subject supported by the National Natural Science Foundation of China (Grant No. 51171156) and the Fundamental Research Funds for the Central Universities of Ministry of Education of China (Grant No. XDJK2014C008).

\section{References}

1. D. J. Lloyd, Particle reinforced aluminium and magnesium matrix composites, Int. Mater. Rev. 39 (1994) 1-23.

2. P. Divecha, S. G. Fishman, S. D. G Karmarkar, Silicon carbide reinforced aluminum-a formable composite, J. Met. 33 (1981) 12-17.

3. A. Ibrahim, F. A. Mohamed, E. J. Lavernia, Particulate reinforced metal matrix composites-a review, J. Mater. Sci. 25 (1991) 1137-1156.

4. T. F. Klimowicz, The large scale commercialization of aluminium-matrix composites, J. Met. 46 (1994) 49-53.

5. E. J. Lavernia, R. J. Perez, J. Zhang, Damping behavior of discontinuously reinforced ai alloy metal-matrix composites, Metall. Mater. Trans. A 26 (1995) 2803-2818.

6. C. Zener, Effect of Strain rate upon plastic flow of steel, J. Appl. Phys. 15 (1944) 22-32.

7. V. V. Polyakov, A. N. Alekseev, Porosity dependence of the internal friction and elastic characteristics of iron powder', Powder Metall. Met. C. 33 (1995) 192-193.

8. N. P. Cheng, S. M. Zeng, Z. Y. Liu, Preparation ,microstructures and deformation behavior of $\mathrm{SiCp} / 6066 \mathrm{Al}$ composites produced by PM route, J. Mater. Proc. Tech. 202 (2008) 27-40.

9. G. Cannelli, F. M. Mazzolai, An investigation on the Ta-H system by internal friction measurements, Nuovo Cimento B. 64 (1969) 171-180.

10. H. Gu, Effect of different heat treatment process on damping peak of $6061 \mathrm{Al} / \mathrm{SiCp}$ MMC produced by spray codeposition, Trans. Nonferrous Met. Soc. China. 12 (2002) 960-966.

11. Granato, K. Lucke, Theory of mechanical damping due to dislocations, J. Appl. Phys. 27 (1996) 583-593.

12. Y. Torisaka, S. Kojima, Superplasticity and internal friction of a superplastic Zn-22\%Al eutectoid alloy, Acta Metall. et Mater. 39 (1991) 947-954.

13. Y. S. Kê, Stress Relaxation across Grain Boundaries in Metals, Phys. Rev. 72 (1947) 41-46. 